\title{
Perygee-Syzygy Tides in the Atmosphere
}

\author{
Nikolay Sidorenkov ${ }^{1 *}$ and Yuri Perevedenzev ${ }^{2}$ \\ ${ }^{1}$ Hydrometeorological Center of Russia, Moscow \\ ${ }^{2}$ Kazan (Volga region) Federal University, Moscow
}

\author{
*Corresponding author: Nikolay Sidorenkov, Hydrometeorological center of Russia, \\ Moscow.
}

\author{
Received Date: April 22, 2019 \\ Published Date: May 06, 2019
}

\section{Opinion}

The years 2016/2017 marked the most striking manifestation of 206-day lunar cycles in weather over European Russia. The smoothed air temperature anomalies followed the variations in the perigee distance (that is, the distance between the Moon and the Earth at times when the Moon is at the nearest point to the Earth, i.e., at the perigee), which represents a sinusoid with a period of 206 days [1]. The 206-day cyclicity results from beats produced by interference of close frequencies of the anomalistic month (27.55 days between two successive perigees) and the synodic month (29.53 days between two successive new moons). This phenomenon is easy to observe by comparing the durations of full moons and new moons [2] or by keeping track of variations in the height of spring tides, i.e., tides at syzygies (at times of new and full moons) [1,3].

The main feature of perigee-syzygy tides is that the curve for each syzygy is well approximated by a sinusoid (beat envelope) with a period of 412 days, and their phases differ by $180^{\circ}$ [1] (Figure 1). Therefore, for any tidal characteristic, every positive anomaly at one syzygy corresponds to a negative anomaly at the next syzygy. The cause is that the orbit of the Moon is elliptic. If a full moon coincides in time with perigee, the distance from the Moon to the Earth is reduced to the minimum and the tidal force becomes maximum (positive anomalies). Roughly in a half-month, at a new moon, the Moon reaches an opposite point of the orbit, i.e., it is at the maximum distance from the Earth (near apogee) and the tidal force becomes minimum (negative anomalies). In 27.55 days, the Moon returns to the perigee, but it happens two days earlier than full moon. Next time, it returns four days earlier, and so on. With every lunar revolution, the tidal force decreases at full moon and increases at new moon and, after 3.5 synodic months, the spring tides at full and new moon become of equal size (at a beat node) and then the tidal force at new moon dominates that at full moon, reaching the maximum range (at a antinodes or crest) after
7 synodic months. Next, the range again decreases up to the next node, which occurs in 3.5 months, and so on. A change in the sign of the anomalies from full to new moons and back always happens at beat nodes in 206 days.

The appearance of a 206-day cycle in the 2017 weather prompted our study of spring tides in the atmosphere, which should be exhibited in oscillations of atmospheric pressure in a similar manner to the spring tides in Figure 1 [1]. Specifically, a series of three-hour ground-surface pressure measured at the VDNKh weather station in Moscow over 2016-2017 was used to calculate daily mean pressure values and their anomalies. The daily pressures at the Moscow State University meteorological observatory averaged over 1966-2010 were used as normals [4]. Next, for each full moon day, we chose the daily mean pressure anomaly over this day. A series of atmospheric pressure anomalies for all new moons was separately generated in a similar manner. With the help of Excel, the atmospheric pressure anomalies at the VDNKh weather station were plotted for full moons (open circles on the broken curve) and for new moons (solid circles) over 20162017 (Figure 1). The diagram of the perigee-syzygy fluctuations of pressure according to observations of the Kazan federal university. Figure 2 has been constructed precisely also later (Figures 1,2).

Figures 1 \& 2 shows that the perigee-syzygy oscillations of atmospheric pressure are highly noisy as compared with the perigee-syzygy sea tides [1,3]. Nevertheless, we can observe the basic features of the atmospheric perigee-syzygy tides, namely, nodes (in the summer of 2016 and May of 2017) and antinodes (in February-March and September-October of 2017). It can be seen that the pressure anomalies at new and full moons are of different signs. The pressure anomalies at full and new moons can be approximated by sinusoids with beat envelope periods of about 412 days and with opposite phases. The range of the oscillations at antinodes reaches $40 \mathrm{mb}$, which is comparable in order of magnitude 
with perigee-syzygy tides in the ocean [1,3]. The deviation of a sea level on $1 \mathrm{~cm}$ is equivalent to anomaly of atmospheric pressure 1 gPa. Thus, Figure $1 \&$ Figure 2 suggest that the geodynamical forces in 2016/17 were able to overcome the stochastic thermodynamics of the atmosphere and imposed their celestial mechanical tidal rhythm on the weather evolution and the variations in pressure and temperatures in Moscow and Kazan. The striking manifestation of a 206-day lunar cycle in the 2016/2017 weather in Moscow and Kazan was associated with perigee-syzygy tides in the atmosphere. The 206-day cycle of perigee-syzygy tides violated the normal seasonal variations in meteorological characteristics. Violation of a seasonal course of weather and many local records of daily values of meteorological characteristics on the European part of Russia has been connected with it in 2016/2017.

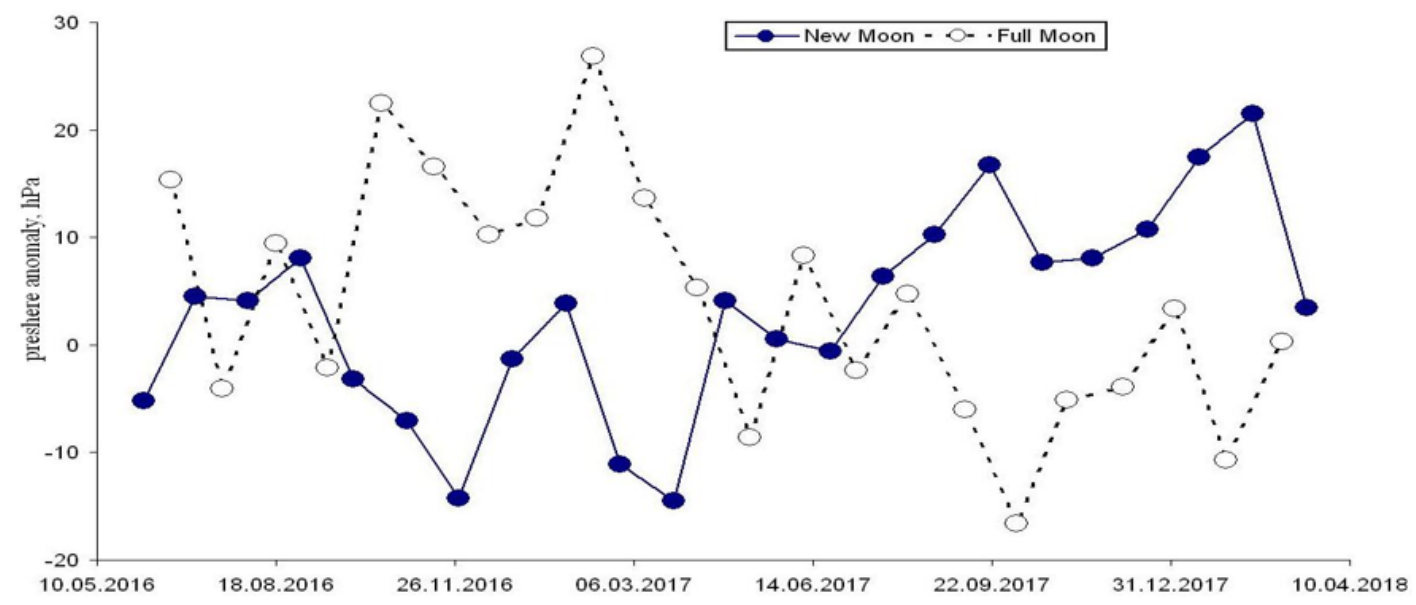

Figure 1: Perigee-syzygy oscillations of the atmospheric pressure measured at Moscow station.

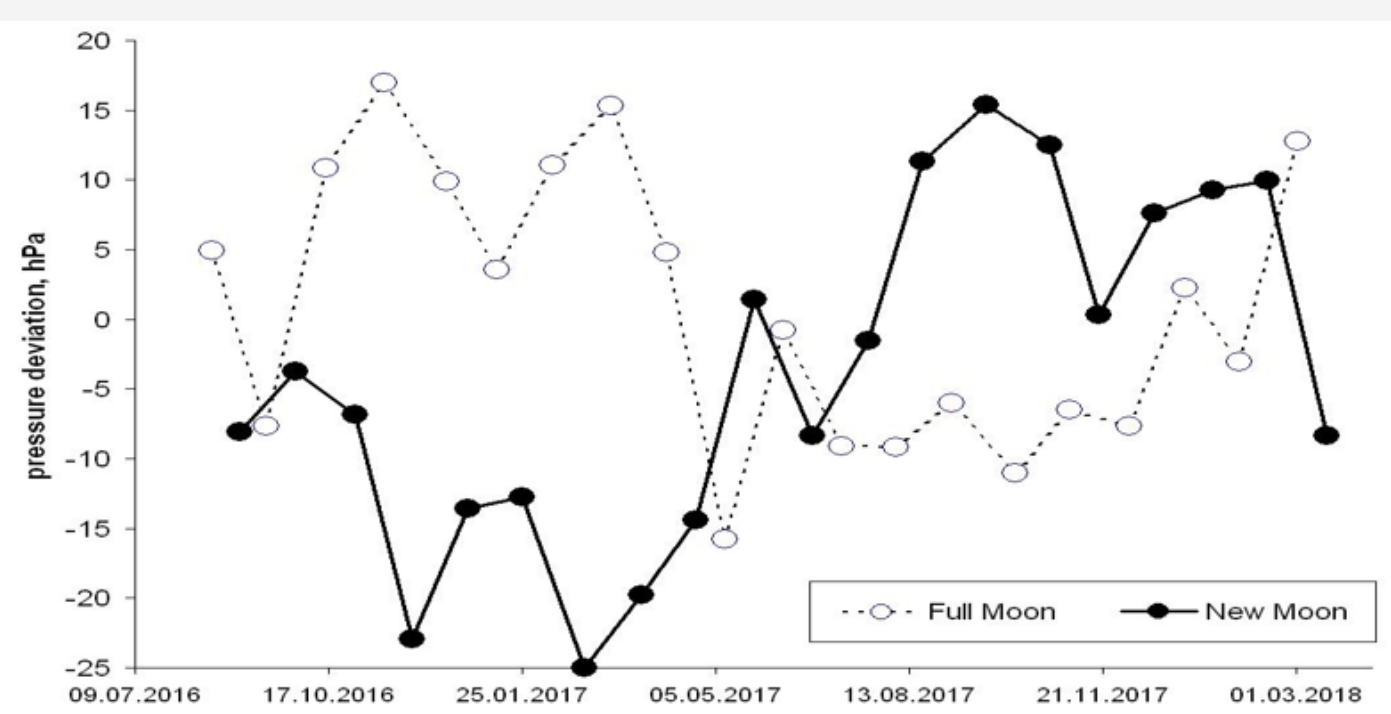

Figure 2: Perigee-syzygy oscillations of the atmospheric pressure measured at Kazan station.

\section{Acknowledgment}

None.

\section{Conflict of Interest}

No conflict of interest.

\section{References}

1. Sidorenkov NS, Chazov VV, Zotov LV, Wilson IRG (2018) The 206-day lunar cycle in weather anomalies. Priroda RAS 4: 19-23.
2. Sidorenkov NS (2016) Celestial Mechanical Causes of Weather and Climate Change. Izvestiya, Atmospheric and Oceanic Physics 52(7): 667-682.

3. Avsuk YuN, Maslov LA (2011) Long Period Tidal Force Variations and Regularities in Orbital Motion of the Earth-Moon Binary Planet System. Earth, Moon, and Planets 108(1): 77-85.

4. Sidorenkov NS, Isaev AA (2008) Thin structure of time variations of atmospheric pressure. Poceedings of Hydrometcenter of Russia 342: 177-186. 